\title{
The Design and Production of Panoramic Micro-Course Video
}

\author{
Tao Men ${ }^{1,}$, , Guihong Zhang ${ }^{2, b}$, Yan Sun ${ }^{3, c}$ and Yuanzhi Wu ${ }^{4, d}$ \\ ${ }_{1,2,4}$ School of Computer Science, Leshan Normal University, Leshan City, China \\ ${ }^{3}$ Finance department of Leshan Normal University, Leshan City, China

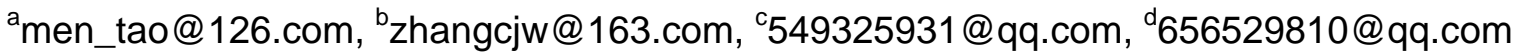

Keywords: MOOC, micro-course video, panoramic video, virtual reality

\begin{abstract}
Massive Open Online Course [1,7] (referred to as MOOC) is currently the hottest online education model. It is mainly composed of many micro-course videos, and has greatly changed the way of learning. But most of the micro-course videos in MOOC are traditional, students will feel boring for a long time, and they do not feel the atmosphere in the traditional classroom. Fortunately, the rapid development of virtual reality technology can greatly improve this situation. This paper proposes a method of making panoramic micro-course video, which is produced by virtual reality technology. In the paper, the immersive panoramic micro-course video production methods and steps will be described in detail. Through panoramic micro-course video, students can immerse themselves in the learning environment to improve learning efficiency.
\end{abstract}

\section{Introduction}

MOOC and SPOC (an abbreviation for Small Private Online Course [2,3,8]) are the representative of the online course, which greatly changed the way of learning. More and more students have changed the habit of learning in fixed time and fixed classroom. Instead, they use the fragmented time to learn with smart mobile device anytime and anywhere. Whether MOOC or SPOC, its main content is composed of many micro-course videos, the quality and performance of micro-course videos greatly affect the learning effect. Unfortunately, there is still a lack of micro-course video that is appealing to students.

Virtual reality (VR) is a computer technology that uses headsets, sometimes in combination with physical spaces or multi-projected environments, to generate realistic images, sounds and other sensations that simulates a user's physical presence in a virtual or imaginary environment $[4,6]$. Using virtual reality technology to show the panoramic video, users can feel that they are fully integrated into the video display environment and have a very real immersive experience. However, the current panoramic video mainly focuses on film and television, entertainment games and so on.., the application in the field of education is scarce.

The application of virtual reality technology in online courses, that it is to create a panoramic micro-course video, can greatly change the students' experience in the online learning. They can fully feel the immersive learning environment to enhance learning interest and improve learning efficiency. The micro-course videos based on VR bring students not only a new learning experience, but also a new visual experience. Further, it is difficult for those traditional videos to display specific teaching content, but it is perfectly presented in panoramic and 3D videos. Though people rarely watch these kinds of videos now, as time goes, they may develop creative ways to take full advantage of the new medium [5].

This paper will show you how to make the panoramic micro-course video for the teaching with the existing conventional hardware and software equipment. It will be useful for the teachers without panoramic video production experience to make panoramic micro-course video successfully. 


\section{The creation process of panoramic micro-course video}

According to the order, design of panoramic micro-course video is mainly divided into teaching design, script design, shooting, post-production. In general, the order of these steps cannot be reversed, because the preliminary work is the basis of the post work. It must be strictly implemented; otherwise, it will lead to confusion. The general production process of panoramic micro-course video is shown in Fig 1.

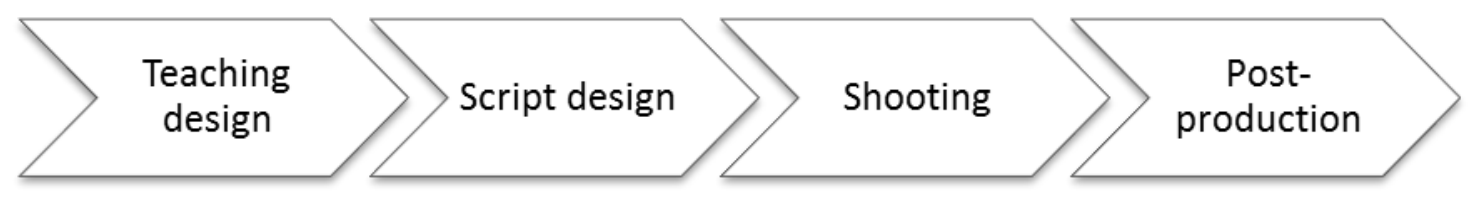

Fig 1 panoramic micro-course video production flow chart

Teaching Design. To produce a good micro-course video, we must first carry out a serious teaching design. Teaching design content also includes a number of aspects: the choice of teaching content, lesson plans, collection of material resources, courseware production and so on.

The choice of teaching content is essential, and the teaching content must be suitable for panoramic video display. In fact, some of the teaching content is not suitable for panoramic video to show. In general, panoramic video is suitable for operability and other environmental-related teaching content. If the teacher wants to show the structure of the student, the panoramic video will bring more immersive feel than the ordinary flat video, on the contrary, if the teacher wants to deduce the mathematical formula for students, panoramic video will not be better than the traditional flat video.

After choosing the right teaching content, we should design the lesson plan. In addition to teaching focus, teaching difficulties, teaching process design, the lesson plan of panoramic micro-course video should also consider the characteristics of panoramic video. We should consider: The teaching site is indoors or outdoors? Is there any student participation in teaching? Does teacher need to move around? Is there a blackboard or PowerPoint? If there are some students in the classroom and the teacher will show the content in PowerPoint, it is better to the teacher do not move around a lot in the teaching process, at the same time, the teacher should design some interaction with the students. Because the indoor space is relatively small, the teacher's movement easily lead to panoramic video stitching failed, in contrast, the interaction between teachers and students, just right can reflect the panoramic video unparalleled advantages: video viewers can freely turn around to see the interactive scene.

Script Design. After designing the lesson plan, we need to design the shooting script according to the lesson plan. This stage is very important for the successful shooting of panoramic video. It will take a lot of time to consider the teaching content and develop a detailed shooting plan.

Because the panoramic video shooting method is different from the traditional video, its script also needs special design. For example: there is not long shot, full shot, medium shot and close shot in panoramic video, so we cannot show the details and focus content by zoom in and zoom out, and the actors or actresses only change their size in the panoramic video by approaching or away from the camera, if they are too close to the camera, panoramic video stitching is difficult to achieve. When we design the script, it should not be through the actor's expression to display the teaching content, but through the sound to pass information. At the same time, the action of actors is also very important, good movement can make panoramic video immersion effect doubled. In general, most of the panoramic videos use mainly fixed lens, the actor can move slowly in a small range, around the camera, or change the distance to the camera. All of these scripts are designed to show better immersion effects.

In the stage of the script design, we also consider the following aspects from the student's perspective: What can students see? Is there any interference information in the video? Will students be attracted to other information without paying attention to teaching content? Different from the ordinary video, panoramic video can show $360^{\circ}$ (horizontal) and even $360^{\circ}+180^{\circ}$ (horizontal and 
vertical) angle of view. Panoramic video display range is shown in Fig 2. In the panoramic video, there is no front and back, also, there is no primary and secondary points, Students' attention must be attracted to the right place, and if they occasionally look around, the panoramic video can also attract their attention back by voice, picture, text, and so on , which is the biggest challenge to script design.

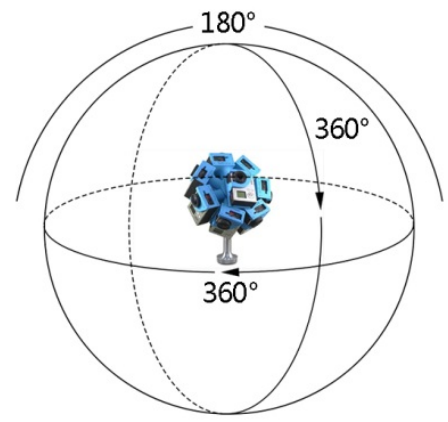

Fig 2 panoramic video display range

The transition between the two panoramic videos is particularly sensible, so you'd better try to use long shot and do not use the teleprompter, because the teleprompter will also be shooting into the video.

Shooting. Panoramic video is 360-degree range of video, which is shot with multiple cameras at the same time. Each camera shoots a scene and they are finally stitched into a complete panoramic video, as if the viewer exposure to the same scene. There are different price of panoramic shooting equipment, their price from tens of thousands to hundred dollars: NextVR, OZO, IM360, Ladybug5, Ista360, Eyesir, 360fly, Theta S, etc. Taking into account the price, performance and other factors, the multiple-Gopro program is currently a better choice. The different panoramic shooting equipment is shown in Fig 3.

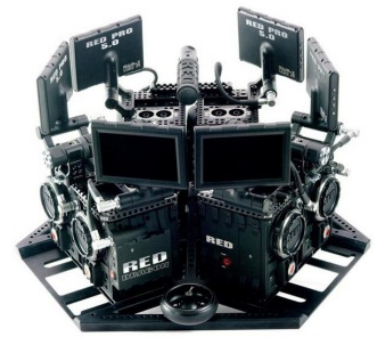

a)NextVR

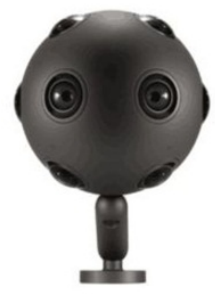

b) $\mathrm{OZO}$

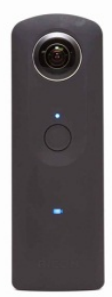

c)Theta $\mathrm{S}$

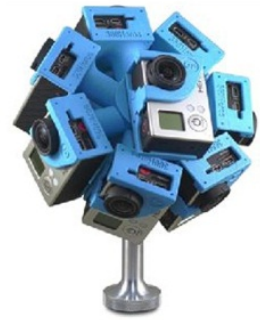

d)Multi-Gopro

Fig 3 the different panoramic shooting equipment

All of this panoramic shooting equipment can be divided into two types: direct imaging and post-stitching imaging. The direct imaging equipment can output panoramic video directly from the hardware. It is easy to use, but it is difficult to expand according to user needs. The post-stitching imaging equipment use multiple cameras at the same time independent shooting video, and all the videos are stitched with software in computer. This process requires a high level of technology, but the video quality can be guaranteed. This paper will focus on production methods of post-stitching imaging.

The post-stitching imaging equipment is usually assembled with 6-20 separate cameras. For the time being, it is usually assembled with multiple Gopro-cameras or other cameras to build a panoramic shooting system. In general, the more cameras you use, the easier the panoramic video is stitching, the higher the video quality is, and the more expensive it is. For the panoramic micro-course, it is enough with 6-8 cameras to shoot the panoramic video, and the video can easily achieve $4 \mathrm{~K}$ quality.

Synchronization among cameras is especially important when multiple cameras are shooting. There are usually synchronizers to ensure that all cameras work at the same time. Each camera should 
use the same settings: exposure, white balance, focal length. If you use 6-8 camera shooting system, you should try to use wide-angle shooting to cover the entire $360^{\circ}+180^{\circ}$ space range.

When we take the video, all the other people and things have to exit the shooting range, only the actors and the necessary props can appear in the video. That is, the director, photographer and other staff member cannot directly see the shooting camera. There are not teleprompter, reflector, fill light, monitor, camera arm, track and other equipment, which are often used in the traditional video shooting process. The cameras usually are fixed on a tripod to shoot with a wireless microphone, and there is no one reminds the actors. Therefore, before the official shooting, the actor should practice and remember the lines, so as to ensure the success rate of shooting.

Post-Production. After all the videos shooting, the post-production will still take a lot of time. First of all, all the videos exported from the cameras must be renamed, and you can rename the videos with the machine number + video number. For example, the first video of the first camera can be renamed 1-01, the second video of the second camera can be renamed 2-02, and so on. At the same time, you have to check the video content carefully to ensure that the same scene shooting video with same time is the same video number.

There are some panoramic video stitching software can be used for post-production: Kolor Autopano Video pro, VideoStitch, Surround360, etc. These software almost can automatic stitch panoramic video, but the details of the videos adjustment still need human being. If there is a gap in the video, or the screen appears overlapping phenomenon, human being need to manually select the stitching point, and then fine-tuning through the computer. In general, the sky is stitched well, but the actors and the object close to the camera need to carefully adjust. Because the panoramic camera can shoot full space, the tripod also will appear in the panoramic video, usually, it will be sheltered by a LOGO picture. In this way, it not only protects the video copyright, but also can solve the panoramic video flaws.

Once video is stitched, you can output the panoramic video. The output video size is typically 2: 1 spherical projection video. On the current stage of the hardware and software conditions, it is not recommended to output more than $4 \mathrm{~K}$ video, because it may not be able to play smoothly. Before becoming a panoramic micro-course video, panoramic video also need to import Edius, Adobe Premiere, Adobe After Effects and other non-linear editing and mix software to add text, illustrations, PowerPoint, animation and other teaching content. It should be noted that the shape of added contents will be deformed, so it is recommended to add to the middle of the panoramic video, this deformation is small and easy to control. Finally, it is added the head leader, tail leader, subtitle then is outputted, a panoramic micro-course video is really completed.

\section{Panoramic micro-course video playback}

If you want to play panoramic micro-course video, there are two main ways. Correspondingly, there are different platforms with hardware and software conditions, and the final effect is also different.

The first way is to play on a PC or website. People can either install a panoramic video player on a PC or visit panoramic website to watch them. In this way, a small part of the panoramic video are showed in player or website, and the user can drag the mouse to change the viewing angle to experience the panoramic effect. These panoramic video Player are VR Player, Kolor Eyes, Gopro VR Player etc. There are also many panoramic websites, and YouTube.com and Youku.com are famous.

The second way is to watch through the VR device. In this way, people need wear a VR helmet to watch the panoramic video. For VR helmet, there are a variety of options: Oculus Rift, PlayStation VR, HTC vive, Storm Mirror, VRBOX, even you can DIY it according to Google CardBoard. Expensive VR helmet usually provides display and processor, while cheap VR helmet will watch with the smart mobile. VR helmet can feel the user's head movement, the display will show different scenes based on head movement, so the users have a strong sense of immersion, which make them focus on the content of micro-course video. 


\section{Conclusion}

Once virtual reality technology is combined with online education, its advantage is obvious. It can not only enrich the content of virtual reality technology, but also can improve the online learning experience. This paper describes the producing methods and process of panoramic micro-course video in the current hardware and software conditions in detail. This paper also compares the difference between the panoramic micro-course video and the ordinary micro-course video in the production process and the precautions. It is a useful exploration for online education and panoramic micro-course video production.

\section{Acknowledgements}

This work is supported by Key Teaching Reform Project of Leshan Normal University (JG2015-ZD14), Key Research Base for Social Science in Sichuan Province-Sichuan Tourism Development Research Center Project (LYC15-09) and Key Lab of Internet Natural Language Processing of Sichuan Provincial Education Department.

\section{References}

[1] Kaplan, Andreas M.; Haenlein, Michael (2016). Higher education and the digital revolution: About MOOCs, SPOCs, social media, and the Cookie Monster. Business Horizons. 59 (4): 441-50.

[2] Goral, Tim. Make Way for SPOCS: Small, Private Online Courses May Provide What MOOCs Can't. University Business (2013).

[3] Fox, Armando. From MOOCs to SPOCs. Communications of the Acm 56.12(2013):38-40.

[4] Wikipedia, Virtual reality, https://en.wikipedia.org/wiki/Virtual_reality

[5] Guo P J, Rubin R, Rubin R. How video production affects student engagement: an empirical study of MOOC videos[C]// ACM Conference on Learning @ Scale Conference. ACM, 2014:41-50.

[6] Qinping Zhao. Summary of Virtual Reality [J]. Scientia Sinica(Informationis), (2009):2-46.

[7] Wikipedia, Massive open online course, https://en.wikipedia.org/wiki/Massive_open_online_course

[8] Wikipedia, Small private online course, https://en.wikipedia.org/wiki/Small_private_online_course 